\title{
Binding Energy Curves from Nonempirical Density Functionals II. van der Waals Bonds in Rare-Gas and Alkaline-Earth Diatomics
}

\author{
Adrienn Ruzsinszky and John P. Perdew \\ Department of Physics and Quantum Theory Group, Tulane University, New Orleans, Louisiana 70118
}

\author{
Gábor I. Csonka* \\ Department of Inorganic Chemistry, Budapest University of Technology and Economics, \\ H-1521 Budapest, Hungary
}

Received: July 15, 2005; In Final Form: October 5, 2005

\begin{abstract}
Binding energy curves have been calculated for the ground-state rare-gas diatomics $\mathrm{Ne}_{2}$ and $\mathrm{Ar}_{2}$ and for the alkaline-earth diatomic $\mathrm{Be}_{2}$ using the nonempirical density functionals from the first three rungs of a ladder of approximations: the local spin density (LSD) approximation, the Perdew-Burke-Ernzerhof (PBE) generalized gradient approximation (GGA), and the Tao-Perdew-Staroverov-Scuseria (TPSS) meta-GGA. Binding energy curves in reasonable agreement with those constructed from experiment are found from PBE and TPSS, which incorporate inhomogeneity corrections that satisfy the Lieb-Oxford bound and so describe the short-range part of the van der Waals interaction. At large internuclear separation, these functionals produce an exponentially decaying attraction in place of the correct long-range $-C_{6} / R^{6}$. Basis-set and exchange-only effects are also discussed.
\end{abstract}

\section{Introduction and Conclusions}

In this work, we aim to determine how well the binding energy curves of van der Waals-bound diatomics can be predicted from a ladder of nonempirical functionals of increasing sophistication: local spin density approximation, generalized gradient approximation (GGA), and meta-GGA. Empirical functionals can be fitted to the equilibrium properties of raregas dimers, while nonempirical ones can predict such properties from exact constraints. Such functionals are capable of describing the short-range part of the van der Waals interaction, which arises from nonbonded density overlap, but not the long-range part that acts between nonoverlapped densities. We expect that still higher rungs of this ladder can continue to be constructed from lower ones and that ultimately the long-range part of the van der Waals interaction, responsible for important effects in biological molecules and in soft condensed matter, can be grafted onto the nonempirical meta-GGA or its successor.

The binding energy curves of rare-gas diatomics provide a useful first level test for the accuracy of a method in describing van der Waals (vdW) (dispersive) attraction, because the exact curves encompass and link both short- and long-range effects. Dispersion is one of the important intermolecular interactions of organic molecules too. For example, it is responsible for the heats of sublimation of hydrocarbon molecules, and it is important for crystal packing of organic molecules, for hostguest systems, for orientation of molecules on surfaces, for the stacking of nucleic acids in DNA, and for solvent properties of polar and apolar compounds. Unfortunately, accurate calculation of the dispersion interaction is a difficult task, and good results for rare-gas diatomics do not guarantee good results for the intermolecular interactions of organic molecules (vide infra). Consequently empirical fitting of density functionals to describe weak interactions in rare-gas diatomics does not necessarily lead to improved results for other molecules. It is known that the
Hartree-Fock (HF) method cannot evaluate dispersion interaction, as it is a pure electron correlation effect. High-level, expensive treatment of electron correlation, typically CCSD(T), and large basis sets, typically aug-cc-pVQZ, are required to evaluate the dispersion interaction accurately. These calculations require expensive computational resources even for relatively small molecules and can be applied only to benchmark studies of small systems.

Generalized gradient approximation (GGA) and meta-GGA density functional theories (DFT) are considerably less demanding of computational resources than the $\operatorname{CCSD}(\mathrm{T})$ methods. Such functionals can at best provide an estimate of the bonding between weakly overlapped densities but can never describe the $-C_{6} / R^{6}$ interaction of nonoverlapped atoms or the related long-range interactions of other nonoverlapped densities, for which fully nonlocal functionals ${ }^{1}$ or generalizations of the random phase approximation ${ }^{2}$ are more promising. It has been shown that some GGA density functionals can describe the short-range part of the interaction for rare-gas diatomics, ${ }^{3-5}$ as can hybrid functionals., ${ }^{6,7}$ However, the BLYP and B3LYP functionals fail to bind the rare-gas diatomics, as demonstrated in refs $4,5,8$ and 9 . In recent work, ${ }^{10}$ the capability of the nonempirical TPSS meta-GGA ${ }^{11}$ and of the hybrid TPSSh $^{12}$ density functionals to predict bond lengths, binding energies, and harmonic vibrational frequencies was tested for the 10 raregas diatomics with $Z \leq 36$. It was shown that both TPSS and TPSSh density functionals produce vdW bonds in rare-gas diatomics and effectively correct the serious overbinding tendency of LSD. ${ }^{9,10}$ Ref 10 also observed that the overcorrection of LSD by the TPSS meta-GGA, resulting in too long bond lengths and too small binding energies, suggests the need for some long-range $\left(1 / R^{6}\right) \mathrm{vdW}$ interaction correction.

In the construction of the TPSS ${ }^{11}$ and PKZB meta-GGAs, ${ }^{13}$ the large-gradient behavior of the nonempirical $\mathrm{PBE}^{14}$ was 
preserved (but more completely in TPSS than in PKZB), since there was no reason to change it and since this choice might preserve the good PBE description of weak (vdW and hydrogen) interactions. However, ref 10 observed that TPSS produces a slightly weaker vdW binding than PBE GGA, despite the same large-gradient behavior. It has been observed that PKZB gives a very poor description of hydrogen bonds, ${ }^{15}$ while TPSS yields a considerably better description of hydrogen bonds. ${ }^{12}$ These results show that the large-gradient behavior of the exchange part of a GGA does not alone determine whether weak bonds with density overlap will be described reliably. This observation refines the suggestion of Zhang et al. ${ }^{4}$

There are now many confirming tests ${ }^{10-12,16-20}$ of the PBE GGA and the TPSS meta-GGA, but there have been few if any such tests for molecular binding energy curves. Calculations of bond length, binding energy, and vibrational frequency test the quality of the energy surface at and near the equilibrium geometry but do not necessarily test its quality under finite expansions and compressions of the bond lengths. We have provided such tests for shared electron bonds ${ }^{21}$ (paper I), and we provide similar tests here for the van der Waals bonds of $\mathrm{Ne}_{2}, \mathrm{Ar}_{2}$, and $\mathrm{Be}_{2}$. We also carry out a basis-set study for the rare-gas diatomics, to show the basis-set effects, and we suggest an optimal basis set for weak-interaction calculations with DFT functionals.

In summary, we have here computed binding energy curves for $\mathrm{Ne}_{2}, \mathrm{Ar}_{2}$, and $\mathrm{Be}_{2}$ with several nonempirical density functionals, including the new TPSS meta-GGA. The conclusions of this and earlier work may be summarized as follows:

(1) Small basis sets without diffuse functions can produce severe overbinding of weakly bound systems, ${ }^{22,23}$ but the addition of diffuse basis functions fixes much of this error. For converged weak-interaction energies considerably larger basis sets (e.g., aug-cc-pVTZ or QZ) are required for GGA or metaGGA than for strong covalent bonds. Even these large basis sets give poor binding energy curves without diffuse functions.

(2) The vdW bond of a diatomic system can be dominated by the short-range part of the vdW interaction and is thus amenable to description by a GGA or meta-GGA. This is so not only for the rare-gas diatomics ${ }^{3,4,10}$ and $\mathrm{Be}_{2}{ }^{24}$ but also for the triplet excited state of $\mathrm{H}_{2}{ }^{21}$ and for $\mathrm{Mg}_{2},{ }^{25,26} \mathrm{Ca}_{2}, \mathrm{Mn}_{2}$, and $\mathrm{Zn}_{2} \cdot{ }^{20}$ But the vdW interaction between layers of atoms, which can include a significant long-range part, can be seriously underestimated by a GGA or meta-GGA. ${ }^{1,2,27-29}$ An example is the benzene dimer, which in its sandwich and T-shaped configurations is van der Waals-bound in a $\operatorname{CCSD}(\mathrm{T})$ calculation. ${ }^{30}$ In a PBE GGA calculation, we have found that it is essentially unbound even with a counterpoise correction. ${ }^{31}$ These results show that a model performing well on the rare-gas diatomics does not necessarily perform well for larger stacking complexes. The important consequence of this is that inclusion of rare-gas diatomics into the DFT training set for empirically fitted density functionals does not guarantee an improvement for larger stacking complexes of chemical or biological interest. The good results for rare-gas diatomics are necessary but not sufficient.

(3) While some empirical GGAs such as BLYP fail to bind the rare-gas diatomics, ${ }^{4,5,9}$ the nonempirical PBE GGA and TPSS meta-GGA describe the short-range part of the vdW interaction sufficiently well to bind them. ${ }^{3,4,10}$ This is largely a consequence of the large-gradient behavior ${ }^{4}$ of the nonempirical functionals, which is chosen to ensure satisfaction of the LiebOxford lower bound on the exchange-correlation energy for all possible electron densities. ${ }^{11,14}$ While the predicted bond lengths and dissociation energies are reasonable estimates, the trend of the experimental dissociation energies (which increase strongly with the nuclear charge $Z$ ) is not reproduced by PBE or TPSS. ${ }^{10}$

(4) Nonempirical GGAs and meta-GGAs tend to overbind those van der Waals-bound diatomics that have valence $\mathrm{s}$ electrons (like $\mathrm{He}_{2}$ and $\mathrm{Be}_{2}$ ) and to underbind those that have valence $\mathrm{p}$ electrons (like $\mathrm{Ne}_{2}$ and $\mathrm{Ar}_{2}$ ). At the exchange-only level, the $\mathrm{B} 88 \mathrm{GGA}^{32}$ binds $\mathrm{Be}_{2}$ but not the rare-gas diatomics.

(5) GGAs and meta-GGAs fail to reproduce the long-range part of the vdW interaction, which tends to $-C_{6} / R^{6}$ as $R \rightarrow \infty$. Unsurprisingly, these approximations produce an attraction that decays exponentially (like the density overlap) when $R \rightarrow \infty$. As expected from their similar large-gradient behaviors, PBE GGA and TPSS meta-GGA are closely similar at large $R$. The local spin density approximation (SVWN5) strongly overbinds the rare-gas diatomics, while the GGAs and meta-GGAs can over- or underbind.

(6) While it is proper for GGAs and meta-GGAs to describe the short-range part of the vdW interaction, a consistent description of the vdW attraction requires a different treatment of the long-range part. ${ }^{33-36}$

(7) The TPSS binding energy curves in $\mathrm{Ne}_{2}$ and $\mathrm{Ar}_{2}$ are somewhat too repulsive at small internuclear distance $R$, and this accounts for the tendency of TPSS to bind less strongly than PBE. This could be favorable for the addition of a damped attractive long-range correction. ${ }^{33-35}$ However, the TPSS overbinding in $\mathrm{Be}_{2}$ is not favorable for such a correction.

(8) At the exchange-only (no correlation) level, exact exchange does not bind $\mathrm{Ne}_{2}$ and $\mathrm{Ar}_{2}$ but exchange-only $\mathrm{PBE}$ or TPSS does bind them. Although some have found this disturbing (e.g., ref 37), it is not so different from the situation for covalent bonds: the GGAs and meta-GGAs model exchange and correlation together much better than they model either alone. This is a consequence of the fact that the exact exchangecorrelation hole is more short-ranged than the exact exchange hole in a molecule. The description of the exchange energy clearly improves up the nonempirical ladder from LSD to PBE to TPSS.

\section{Methods}

We performed calculations with the LSD or Slater, B88, ${ }^{32}$ PW91, ${ }^{38} \mathrm{PBE}^{14}$ and TPSS ${ }^{11}$ DFT functionals and the CCSD(T) method with a series of increasing-quality basis sets, from 6-31G(d) to aug-cc-pVQZ. ${ }^{39}$ For the calculations we used the Gaussian 03 program package. ${ }^{40}$ For the atoms we used tight convergence criteria ( $\mathrm{scf}=$ tight). The binding energy curves were calculated with ultrafine integration grid sizes (99 radial, 590 angular points). With this grid, some functionals predicted binding energy curves with many small oscillations. To decrease the numerical noise and obtain a smooth binding energy curve at larger internuclear distances, grids containing up to 250 radial and 590 angular points were used [integral(grid $=250$ 590)].

We compared the experimental binding energy curves obtained by Ogilvie and Wang ${ }^{41}$ to those proposed by Aziz et al. ${ }^{42,43}$ For $\mathrm{Ne}_{2}$, the difference is less than $0.0004 \mathrm{kcal} / \mathrm{mol}$ for $2.0 \AA<R<6.5 \AA$, but it grows to $0.002 \mathrm{kcal} / \mathrm{mol}$ at $R=9 \AA$. For $\mathrm{Ar}_{2}$, the difference is less than $0.001 \mathrm{kcal} / \mathrm{mol}$ for $3.5 \AA<$ $R<6.0 \AA$, but it grows to $0.1 \mathrm{kcal} / \mathrm{mol}$ at $R=8 \AA$ and continues to grow to infinity at larger $R$, since only the analytic form of Aziz et al. properly tends to zero as $R$ tends to $\infty$. Thus we shall take the binding energy curves of Aziz et al. as our standard for the rare-gas diatomics. 
Ne-Ne with PBE

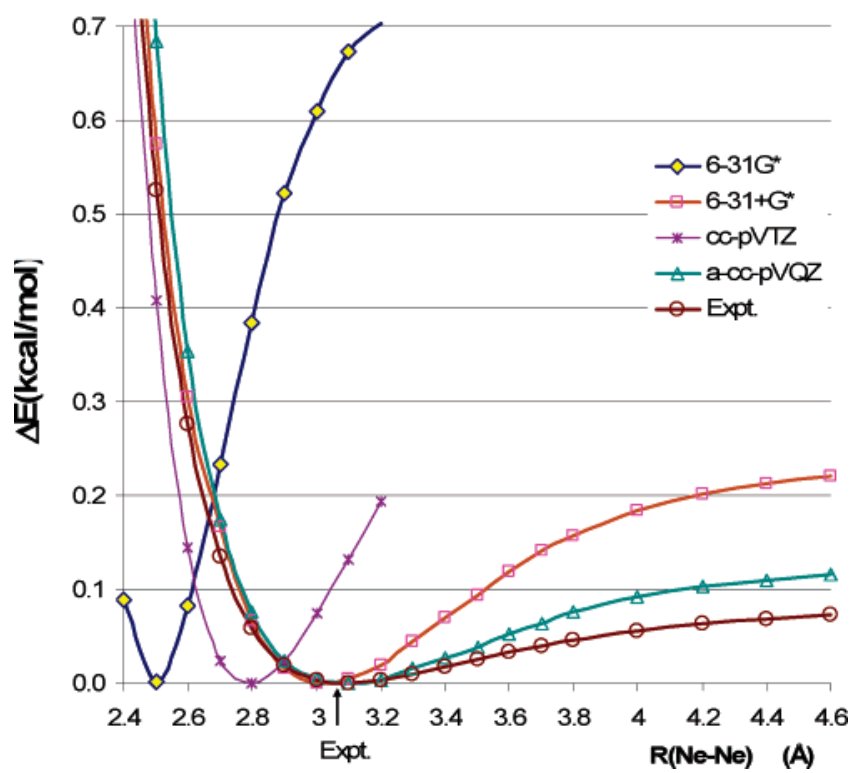

Figure 1. Experimental (ref 42) and PBE relative potential energy $(\Delta E)$ curves calculated with 6-31G(d), 6-31+G(d), cc-pVTZ, and augcc-pVQZ basis sets for $\mathrm{Ne}_{2}$. An ultrafine (pruned, 99, 590) grid was used in the PBE calculations. The experimental equilibrium distance is marked as Expt. $(1$ hartree $=627.5 \mathrm{kcal} / \mathrm{mol}$; $1 \mathrm{bohr}=0.5292 \AA$. $)$ Note that small basis sets without diffuse functions overbind strongly.

\section{Results and Discussion}

3.a. Basis-Set Effects for Weakly Bound Systems. The energy of the vdW interaction is very small compared with the energies of normally bonded molecules or hydrogen-bonded complexes, so the effect of the basis-set error can be relatively large and may affect the conclusions drawn from calculated results. An earlier study ${ }^{22}$ applied a moderate 6-31G(d,p) basis set to test 25 density functionals on dispersion-bound dimers including species such as $\mathrm{Ar}_{2}$ and $\mathrm{Ne}_{2}$. The results of that study differ considerably from other results obtained in ref 10 and also from those obtained with larger basis sets. ${ }^{23}$

The PBE interatomic interaction potential of the neon dimer was calculated using basis sets of increasing quality from 6-31G(d) to aug-cc-pVQZ and compared to experiment, ${ }^{42}$ as shown in Figure 1. The limitations of the 6-31G(d) basis set have a considerable effect on the curve, making it too attractive and yielding a deep minimum at an artificially short distance, 2.52 $\AA$ (with $D_{\text {e }}$ about $0.7 \mathrm{kcal} / \mathrm{mol}^{22}$ ). However, the PBE/aug-ccpVQZ potential curve has its minimum at $3.10 \AA$. The calculated $D_{\mathrm{e}}$ is $0.121 \mathrm{kcal} / \mathrm{mol}^{10}$ (cf., Figure 2). These values are close to experimental values $\left(3.091 \AA\right.$ and $\left.0.084 \mathrm{kcal} / \mathrm{mol}^{41}\right)$. Figure 1 also shows that adding a diffuse function to the $6-31 \mathrm{G}$ or $6-311 \mathrm{G}$ basis set yields a very much improved potential energy curve at a small computational cost. The effect of the diffuse functions is large for the least expensive 6-31G(d) basis set and small for the very expensive (already diffuse) cc-pVQZ basis set. We note that diffuse functions added to small basis sets are necessary for the large-gradient part of the functional. The performance of the $6-31+\mathrm{G}(\mathrm{d})$ and $6-311+\mathrm{G}(\mathrm{d})$ basis sets makes it possible to use these basis sets for weak-interaction calculations ${ }^{44}$ as quite economic alternatives to the aug-cc-pVQZ basis set. Further basis-set study shows that adding more $\mathrm{d}$ and $\mathrm{f}$ functions to the 6-31G(d) basis set has a minor effect on the $\mathrm{Ne}-\mathrm{Ne}$ distance, in agreement with ref 23. For a proper calculation of $R_{\mathrm{e}}$, small basis sets must be augmented by diffuse functions.

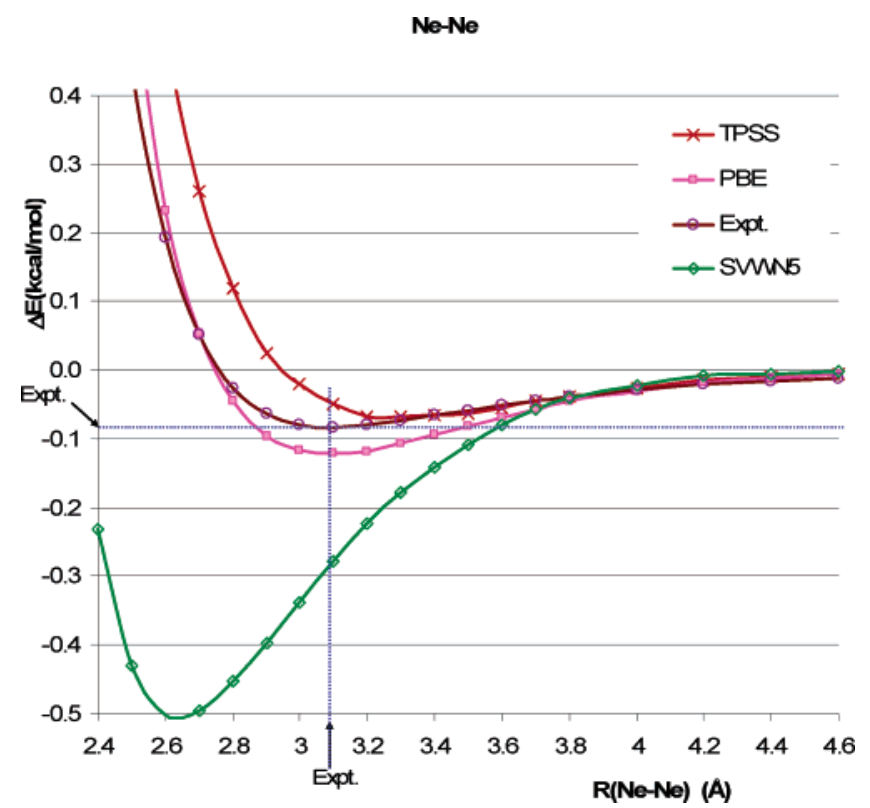

Figure 2. Binding energy $\left[\Delta E=E\left(R_{\mathrm{Ne}-\mathrm{Ne}}\right)-2 E(\mathrm{Ne})\right]$ curves calculated with the aug-cc-pVQZ basis set for $\mathrm{Ne}_{2}$. An ultrafine (pruned, $99,590)$ grid was used in the calculations. The experimental binding energy, $0.083 \mathrm{kcal} / \mathrm{mol}$, and the equilibrium distance, $3.09 \AA$, are marked as Expt. (equilibrium values from ref 41, curve from ref 42). Note that TPSS is more repulsive at small $R$ than PBE is.

It seems to be a general feature of weak bonds that small basis sets overbind in comparison to the basis-set limit. $\left(\mathrm{Be}_{2}\right.$ of section 3.e. is, however, an exception.) We have observed the same effect in PBE calculations for two different configurations of the water dimer, using the small $6-31+\mathrm{G}(\mathrm{d})$ and the large aug-cc-PVQZ basis sets. For these hydrogen-bonded systems, PBE in the large basis set yields excellent results, and TPSS binds a little less strongly than PBE.

3.b. Neon Dimer, with and without Correlation. Figure 2 shows the TPSS, PBE, SVWN5/aug-cc-pVQZ, and experimental binding energy curves for the neon dimer. We also calculated a $\operatorname{CCSD}(\mathrm{T})$ curve with this basis set. We approximated the integrated difference between the experimental ${ }^{42}$ and calculated curves by the sum of the absolute values of $E$ (experiment, $R_{i}$ ) - E(calculated, $R_{i}$ ), where $R_{i}$ is the $i$ th distance in Figure 2. For the repulsive range, $2.5 \AA<R(\mathrm{Ne}-\mathrm{Ne})<3.1 \AA$, these sums are $0.015,0.041$, and $0.185 \mathrm{kcal} / \mathrm{mol}$ for the $\operatorname{CCSD}(\mathrm{T})$, PBE, and TPSS models, respectively; the TPSS/aug-cc-PVQZ curve is too repulsive below the equilibrium internuclear distance $(3.27 \AA)$. For the attractive range, $3.2 \AA<R(\mathrm{Ne}-\mathrm{Ne})<3.8$ $\AA$, these sums are $0.019,0.023$, and $0.005 \mathrm{kcal} / \mathrm{mol}$ for the CCSD(T), PBE, and TPSS models, respectively. For the distant range, $4.0 \AA<R(\mathrm{Ne}-\mathrm{Ne})<4.6 \AA$, these sums are $0.008,0.003$, and $0.005 \mathrm{kcal} / \mathrm{mol}$ for the CCSD(T), PBE, and TPSS models, respectively.

In an earlier paper, ${ }^{10}$ it was observed that PBE yields a more accurate prediction of bond lengths for the rare-gas diatomics than the TPSS functional does. The latter yields systematically longer bond lengths and smaller binding energies. ${ }^{10}$ The TPSS/ aug-cc-PVQZ potential energy curve of Figure 2 explains this by the larger repulsion below the equilibrium internuclear distance. However, it also shows that the attractive and longrange parts of the TPSS curve are better than those of the CCSD(T) or PBE curves for the neon dimer. This is a feature that cannot be observed from the equilibrium distance or the binding energy alone.

We plot the exchange-only binding energy curves for $\mathrm{Ne}_{2}$ in Figure 3 . We compare the repulsive exact exchange (HF) to 
Ne-Ne Ex only

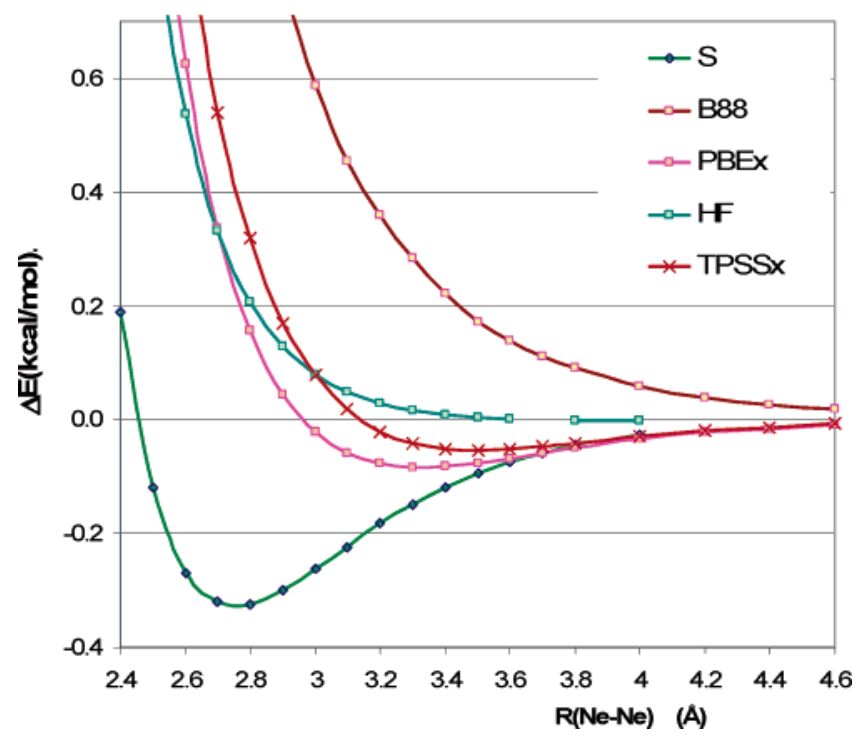

Figure 3. Exchange-only binding energy $\left[\Delta E=E\left(R_{\mathrm{Ne}-\mathrm{Ne}}\right)-2 E(\mathrm{Ne})\right]$ curves for $\mathrm{Ne}_{2}$. The functionals Slater (S), Becke (B88), PBEx, exact exchange (HF), and TPSSx were combined with the aug-cc-pVQZ basis set. An ultrafine (pruned, 99, 590) grid was used in the calculations. Note that TPSSx is closer to HF than PBEx is.

Slater (S), PBE, and TPSS exchange-only functionals, using the aug-cc-pVQZ basis set. We also included two popular exchange functionals, the $\mathrm{B} 88^{32}$ and the PW91. ${ }^{38}$ The B88 exchange is far more repulsive than the HF and fails to bind $\mathrm{Ne}-\mathrm{Ne}$ no matter what kind of correlation functional is used, if the applied basis set is close to the basis-set limit. ${ }^{9,22,45}$ B88 is not a good imitator of HF or exact exchange, even though both are purely repulsive for the rare-gas diatomics. A fairly good imitator of $\mathrm{HF}$ exchange in $\mathrm{vdW}$ bonds seems to be revPBE. ${ }^{1}$

Figure 3 shows the serious overbinding of the $\mathrm{S}$ exchange and its too short $R_{\mathrm{e}}(\mathrm{Ne}-\mathrm{Ne})^{9}$ (cf., the known overestimation of the hydrogen-bond energy by LSDA). The PBE exchange improves considerably over the $\mathrm{S}$ exchange, but it remains slightly binding. Figure 3 shows that the PBE and TPSS exchange curves cross the $\mathrm{HF}$ curve at $\mathrm{R}(\mathrm{Ne}-\mathrm{Ne}) \approx 2.7$ and $3.0 \AA$, respectively. The binding of the PBE exchange is further reduced by the TPSS exchange.

PBE and TPSS work as well as they do near $R_{\mathrm{e}}$ because of a cancellation of error between exchange and correlation, which is also familiar for the atomization energies of covalent molecules ${ }^{17,18}$ and the surface energies of metals. ${ }^{19}$ The systematic improvement up the DFT ladder has been clearly demonstrated for the exchange functionals.

3.c. Argon Dimer. The interatomic interaction potential of the argon dimer was calculated using the aug-cc-pVTZ basis set and compared to experiment, ${ }^{43}$ as shown in Figure 4. The curves show the same tendency observed for the neon dimer in Figure 2. The overbinding of the SWVN5 curve is corrected by the PBE and overcorrected by the TPSS functional $\left(D_{\mathrm{e}}=\right.$ $0.773,0.151$, and $0.072 \mathrm{kcal} / \mathrm{mol}$, respectively, vs the experimental $0.285 \mathrm{kcal} / \mathrm{mol}^{41}$ ). The $R_{\mathrm{e}}$ and $D_{\mathrm{e}}$ that we find for $\mathrm{Ne}_{2}$ and $\mathrm{Ar}_{2}$ in PBE and TPSS using aug-cc-pVQZ are in good agreement with those of Tao and Perdew, ${ }^{10}$ using the same basis set with or without BSSE correction. The hybrid functionals are slightly less binding. Mixing $25 \%$ of the exact exchange decreases the PBE binding energy by $30 \%$, and mixing $10 \%$ of the exact exchange decreases the TPSS binding energy by $11 \%$.

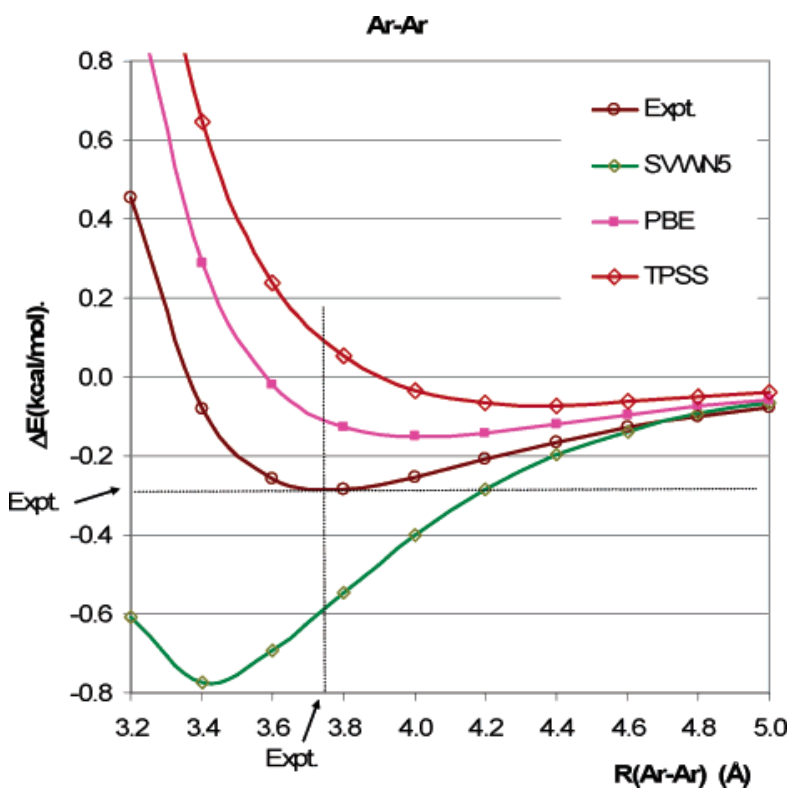

Figure 4. Binding energy $\left[\Delta E=E\left(R_{\mathrm{Ar}-\mathrm{Ar}}\right)-2 E(\mathrm{Ar})\right]$ curves calculated with the aug-cc-pVTZ basis set for $\mathrm{Ar}_{2}$. An ultrafine (pruned, 99, 590) grid was used in the calculations. The experimental binding energy, $0.285 \mathrm{kcal} / \mathrm{mol}$, and the equilibrium distance, $3.757 \AA$, are marked as Expt. (equilibrium values from ref 41, curve from ref 43). As discussed in section 3.c., aug-cc-pVQZ curves are very similar to these. Note that TPSS is more repulsive at small $R$ than PBE is.

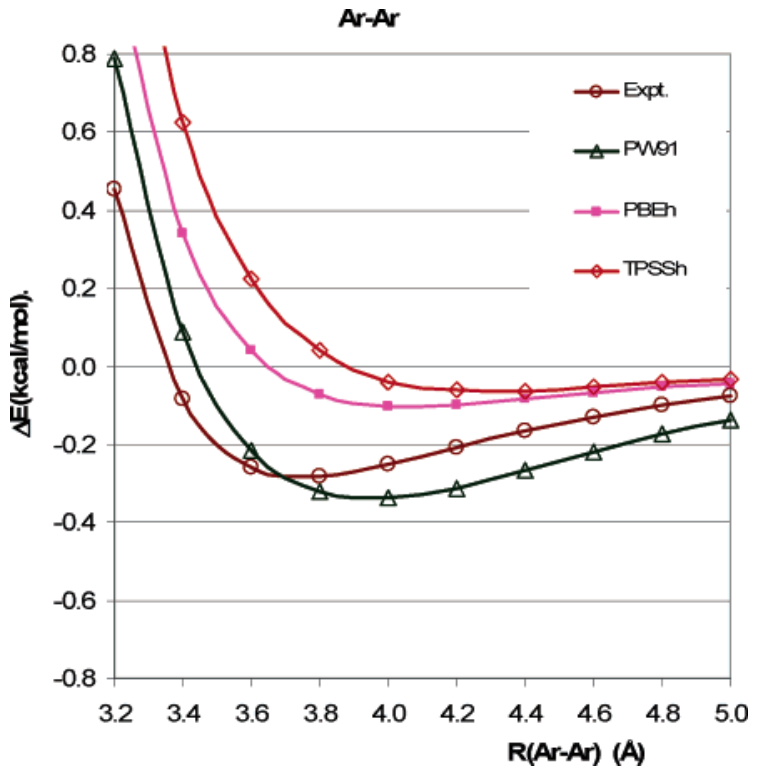

Figure 5. Binding energy $\left[\Delta E=E\left(R_{\mathrm{Ar}-\mathrm{Ar}}\right)-2 E(\mathrm{Ar})\right]$ curves calculated with the aug-cc-pVQZ basis set for $\mathrm{Ar}_{2}$. An ultrafine (pruned, 99, 590) grid was used in the calculations. In the large- $R$ part of this figure, PW91 is deeper than experiment, while PBE of Figure 4 is shallower.

The aug-cc-pVQZ curves (cf., ref 5) and the 6-31+G(d) curves are similar to the aug-cc-pVTZ curves. Summarizing the results obtained for helium (not shown), neon, and argon diatomics: the PBE and TPSS functionals yield a weak binding for raregas diatomics, but they do not follow the increasing experimental binding trend for the helium, neon, and argon dimer series.

The PW91, ${ }^{38}$ PBEh, ${ }^{46,47}$ and TPSSh/aug-cc-pVQZ curves for $\mathrm{Ar}_{2}$ are shown in Figure 5. Figures 4 and 5 show the range of predictions possible from semilocal functionals constructed only from exact constraints. PBE and PW91 are almost identical for reduced gradients $s<3$, and thus for almost any calculated property. However, they differ strongly for $s>3$ (a region for 


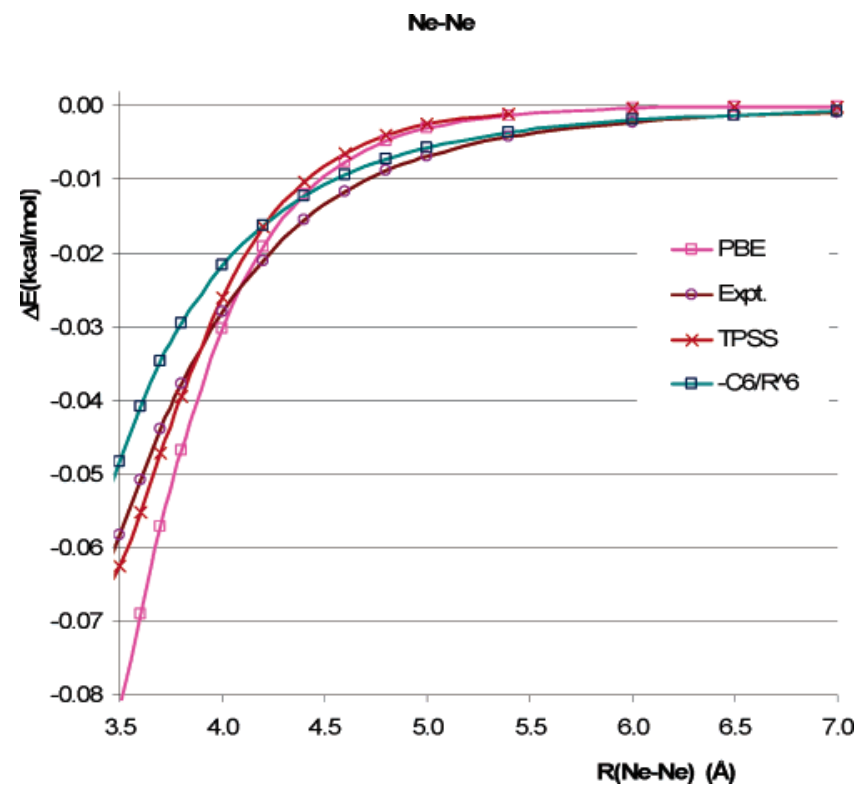

Figure 6. Binding energy $\left[\Delta E=E\left(R_{\mathrm{Ne}-\mathrm{Ne}}\right)-2 E(\mathrm{Ne})\right]$ curves at large internuclear distances calculated with the aug-cc-pVQZ basis set for $\mathrm{Ne}_{2}$, compared to experiment (HFD-B potential) (ref 42) and $-C_{6} / R^{6}$ (ref 42). Grids containing 250 radial and 590 angular points were used. Note that the TPSS and PBE curves are similar at large $R$.

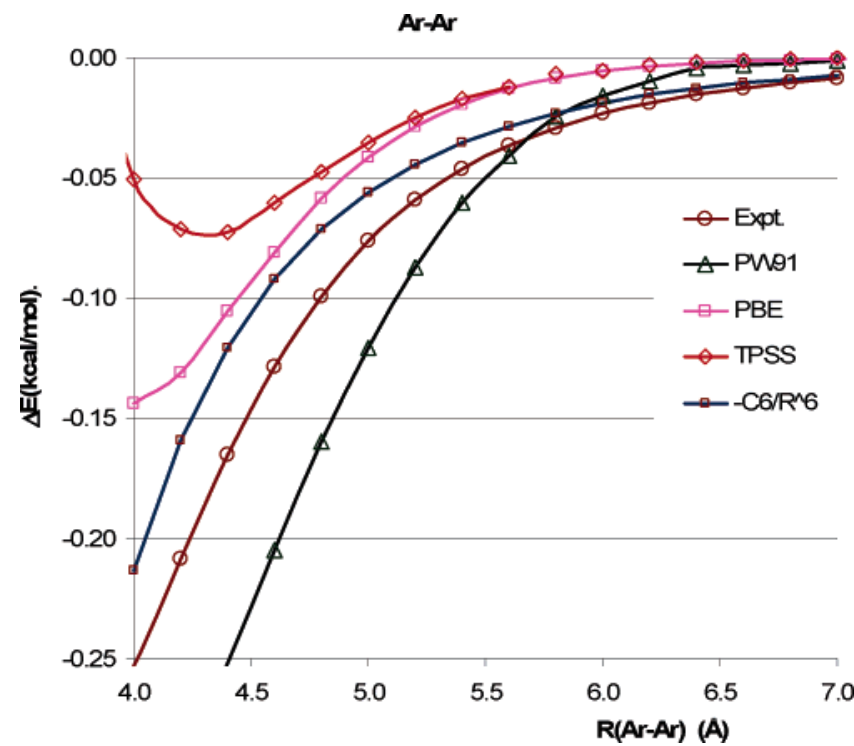

Figure 7. Binding energy $\left[\Delta E=E\left(R_{\mathrm{Ar}-\mathrm{Ar}}\right)-2 E(\mathrm{Ar})\right]$ curves at large internuclear distances calculated with the aug-cc-pVQZ basis set for $\mathrm{Ar}_{2}$ compared to experiment (HDDID1 Ar-Ar potential) (ref 43) and $-C_{6} / R^{6}$ (ref 43). Grids containing 250 radial and 590 angular points were used. Note that the TPSS and PBE curves are similar at large $R$.

which semilocal functionals cannot be reliable), and this difference is significant on the scale of the rare-gas binding curves. It makes the PW91 curve much deeper than the PBE curve in the large- $R$ range of Figures 4 and 5.

3.d. Exponential Decay of Approximate Binding Curves at Long Range. Figures 6 and 7 show the long-range behavior of the GGA and meta-GGA potential energy curves for $\mathrm{Ne}_{2}$ and $\mathrm{Ar}_{2}$, respectively. To obtain correct smooth curves, we had to increase the integration grid size to 250 radial and 590 angular points. We also show the experimental curves with their $-C_{6}$ l $R^{6}$ term, as the latter is dominant for the long-range interaction. For $\mathrm{Ne}_{2}$ the HFD-B ${ }^{42}$ and for $\mathrm{Ar}_{2}$ the HDDID1 potential ${ }^{43}$ were used.

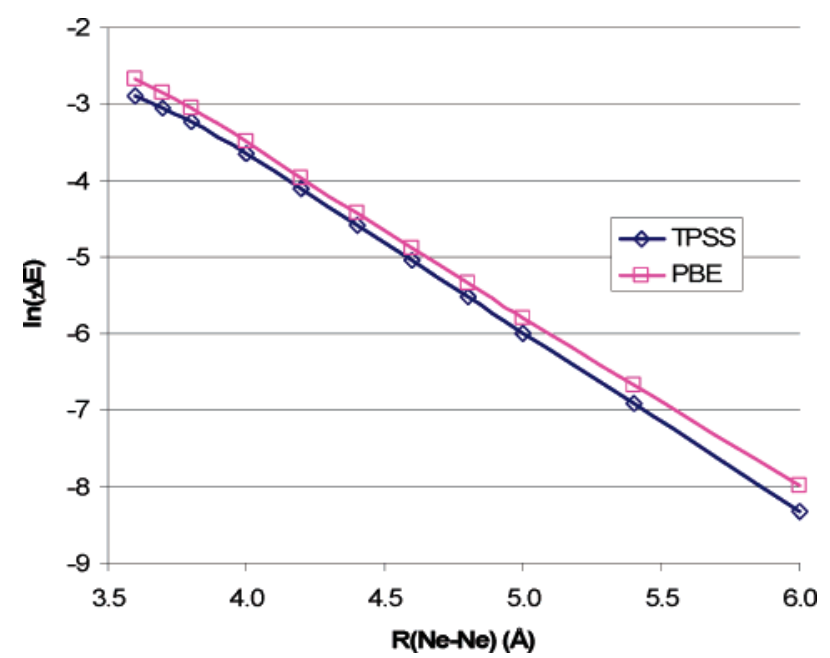

Figure 8. Logarithm of the binding energy $\left[\Delta E=E\left(R_{\mathrm{Ne}-\mathrm{Ne}}\right)-2 E(\mathrm{Ne})\right]$ curves at large internuclear distances calculated with the aug-cc-pVQZ basis set for $\mathrm{Ne}_{2}$. Note the exponential decay of $\Delta E$ at large $R$.

The logarithms of the PBE and TPSS/aug-cc-PVQZ potential energy curves for $\mathrm{Ne}_{2}$ are shown in Figure 8. The electron density of a free atom decays as $\exp (-2 \alpha r)$ as $r \rightarrow \infty$, where $\alpha=\sqrt{-2 \epsilon_{\mathrm{HOAO}}}$ and $\epsilon_{\mathrm{HOAO}}$ is the eigenvalue of the highest occupied atomic orbital. The bond center is at a distance $r=R(\mathrm{Ne}-\mathrm{Ne}) / 2$ from each nucleus, so its density $\rho$ decays as $\exp (-\alpha R(\mathrm{Ne}-\mathrm{Ne}))$. Now

$$
\frac{\mathrm{d}}{\mathrm{d} R} \ln \left[\rho^{4 / 3}\right]=-(4 / 3) \alpha
$$

The PBE and TPSS/aug-CC-pVQZ calculations make $\epsilon_{\mathrm{HOAO}}$ equal to -0.49068 and -0.49640 hartree, respectively. These values yield -1.32 and -1.33 hartree, respectively, for the righthand-side of eq 1 . These values are similar to the derivatives of

$$
\frac{\mathrm{d}}{\mathrm{d} R} \ln [-\Delta E]
$$

shown in Figure 8, -1.19 and -1.24 hartree for PBE and TPSS, respectively. Thus the $-\Delta E$ from Figures 6 and 7 are exponentials with about the same exponent as the asymptotic $\rho^{4 / 3}$.

Because the exact exchange-correlation hole in a molecule is more localized than the exact exchange hole, GGA and metaGGA are better suited to the description of exchange and correlation together than to the description of either separately. Nevertheless, it is of interest to understand how exact exchange and correlation (or dispersion) contribute separately to the vdW bond. A hint can be found in the work of Tang and Toennies, ${ }^{48}$ who make an analytic model of the binding energy curve of a rare-gas dimer in which the repulsive term $\sim \exp (-b R)$ is Hartree-Fock-like and the attractive or dispersion part is $-\left(C_{6}\right)$ $\left.R^{6}\right) f_{6}(R)-\left(C_{8} / R^{8}\right) f_{8}(R)-\left(C_{10} / R^{10}\right) f_{10}(R)$. The dispersion coefficients $C_{6}, C_{8}$, and $C_{10}$ are experimental or theoretically predicted. Figure 9 shows a decomposition of their model for $\mathrm{Ne}_{2}$ in the range $2.7 \AA<R<7 \AA$, in which the damping functions $f_{n}(R)$ remain close to 1 , especially for $n=6$ and 8 . This figure shows that the $-C_{8} / R^{8}$ and $-C_{10} / R^{10}$ contributions are fairly important around the equilibrium bond length.

3.e. Beryllium Dimer, with and without Correlation. Finally, we turn to $\mathrm{Be}_{2}$, a van der Waals-bound diatomic from the alkaline-earth group. Although formally van der Waalsbound, $\mathrm{Be}_{2}$ displays much more density overlap than the raregas dimers do. For quantum chemical wave function methods, 

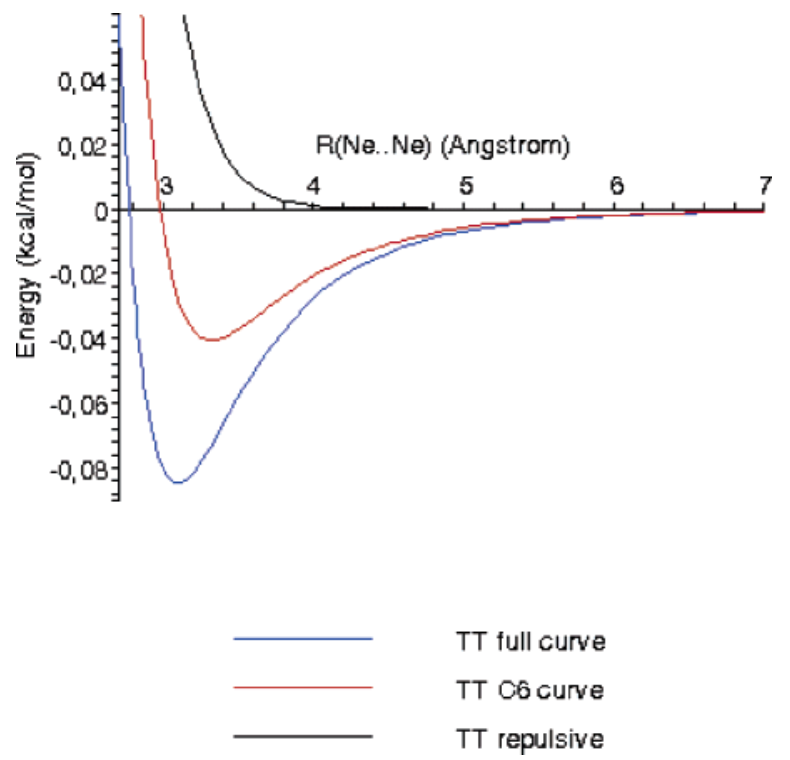

Figure 9. Decomposition of the Tang-Toennies (ref 48) (TT) analytic model for the binding energy curve of $\mathrm{Ne}_{2}$ into a repulsive term and an attractive sum of dispersion terms, as discussed in the text. TT C6 is the damped $C_{6}$ term. The TT full curve includes also damped $C_{8}$ and $C_{10}$ terms as well as the repulsive term. The repulsive term mimics exact exchange.

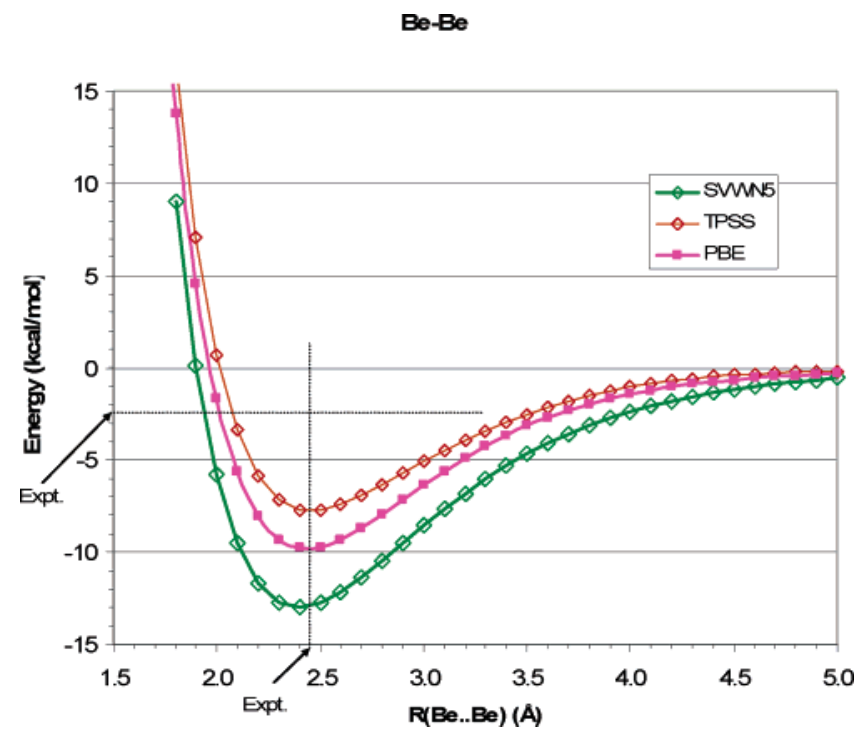

Figure 10. SVWN5, PBE, and TPSS binding energy curves for $\mathrm{Be}_{2}$, using the 6-311G(d) basis set. The experimental binding energy, 2.4 $\mathrm{kcal} / \mathrm{mol}$, and the equilibrium distance, $2.45 \AA$, are marked as Expt. (refs 52 and 53). The relatively small 6-311G(d) basis set is adequate for DFT calculations of $\mathrm{Be}_{2}$, as discussed in section 3.e. Note that TPSS is more repulsive than $\mathrm{PBE}$ at small $R$.

this is a very challenging system. ${ }^{49}$ Comparison of full CI and CCSD curves shows that the CCSD method poorly describes the electron correlation at equilibrium, and very expensive connected quadruple excitations ${ }^{50}$ (e.g., CCSDTQ method) and a large basis $\operatorname{set}^{49,50}$ or multireference $\mathrm{CC}$ methods (e.g., MR$\mathrm{AQCC}-\mathrm{mc})^{49}$ are required for the accurate description of this bond. A full CI calculation yields a binding energy curve with two minima, one of them very shallow. ${ }^{51}$

Figure 10 shows the PBE and TPSS binding energy curves for $\mathrm{Be}_{2}$, calculated with the $6-311 \mathrm{G}(\mathrm{d})$ basis set; the larger 6-311+G(d) or aug-cc-PVTZ basis sets give a very similar curve, yielding a slightly stronger binding (by $0.4-0.5 \mathrm{kcal} /$ mol depending on the functional). The experimental equilibrium bond length ${ }^{52} R_{\mathrm{e}}=2.45 \AA$ is faithfully reproduced by both

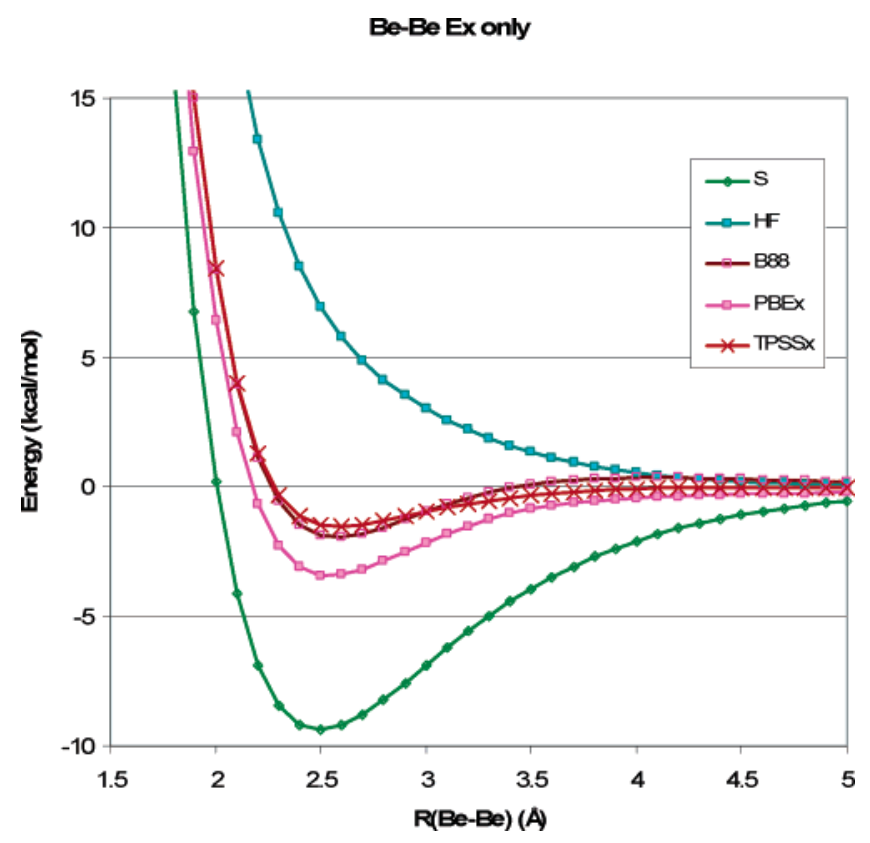

Figure 11. Exchange-only binding energy curves for $\mathrm{Be}_{2}$, using the 6-311G(d) basis set. Note that TPSSx is closer to HF than PBEx is. Note further that B88 binds here (but not in Figure 3) and that it has a spurious maximum at large $R$. The experimental binding energy is $2.4 \mathrm{kcal} / \mathrm{mol}($ ref 53).

functionals, although the experimental dissociation energy $D_{\mathrm{e}}$ $=2.4 \mathrm{kcal} / \mathrm{mol}^{53}$ is overestimated by a factor of $4(\mathrm{PBE})$ or 3 (TPSS). We note that these or similar functionals tend to overestimate the atomization energy when the valence electrons are $\mathrm{s}$ electrons, as in $\mathrm{He}_{2},{ }^{10} \mathrm{Be}_{2},{ }^{24} \mathrm{Mg}_{2},{ }^{25,26} \mathrm{Ca}_{2},{ }^{20}$ and $\mathrm{Zn}_{2},{ }^{20}$ and to underestimate the atomization energy when the valence electrons are p electrons, as in $\mathrm{Ne}_{2}, \mathrm{Ar}_{2}$, and $\mathrm{Kr}_{2}{ }^{10}$

Figure 11 shows exchange-only binding curves for $\mathrm{Be}_{2}$. Note that here, as for the rare-gas diatomics, exact exchange-only is purely repulsive, but now (unlike the rare-gas case) B88 exchange-only is attractive although it shows a spurious repulsive tail above $3.5 \AA$.

Acknowledgment. The first author (A.R.) acknowledges fellowship support from the Pro Progressio Foundation and OTKA under Grant PD050014. The second author (J.P.P.) acknowledges the support of the U.S. National Science Foundation under Grants DMR-01-35678 and DMR-05-01588, and the third author (G.I.C.) acknowledges the support of OTKA under Grant T034764 (Hungary).

\section{References and Notes}

(1) Langreth, D. C.; Dion, M.; Rydberg, H.; Schroeder, E.; Hyldgaard, P.; Lundqvist, B. I. Int. J. Quantum Chem. 2005, 101, 599.

(2) Dobson, J. F.; Wang, J.; Dinte, B. P.; McLennan, K.; Le, H. M. Int. J. Quantum Chem. 2005, 101, 579.

(3) Patton, D. C.; Pederson, M. R. Phys. Rev. A 1997, 56, R2495; Patton, D. C.; Pederson, M. R. Phys. Rev. A 2005, 71, 019906 (erratum)

(4) Zhang, Y.; Pan, W.; Yang, W. J. Chem. Phys. 1997, 107, 7921.

(5) Tsuzuki, S.; Lüthi, H. P. J. Chem. Phys. 2001, 114, 3949.

(6) Xu, X.; Goddard, W. A., III. J. Phys. Chem. A 2004, 108, 8495.

(7) Adamo, C.; Barone, V. J. Chem. Phys. 1999, 110, 6158.

(8) Kristyán, S.; Pulay, P. Chem. Phys. Lett. 1994, 229, 175.

(9) Pérez-Jordá, J. M.; Becke, A. D. Chem. Phys. Lett. 1995, 233, 134.

(10) Tao, J.; Perdew, J. P. J. Chem. Phys. 2005, 122, 114102.

(11) Tao, J.; Perdew, J. P.; Staroverov, V. N.; Scuseria, G. E. Phys Rev. Lett. 2003, 91, 146401 for other meta-GGAs see references therein. (12) Staroverov, V. N.; Scuseria, G. E.; Tao, J.; Perdew, J. P. J. Chem Phys. 2003, 119, 12129; 2004, 121, 11507 (E).

(13) Perdew, J. P.; Kurth, S.; Zupan, A.; Blaha, P. Phys. Rev. Lett. 1999, 82,$254 ; \mathbf{1 9 9 9}, 82,5779$ (E). 
(14) Perdew, J. P.; Burke, K.; Ernzerhof, M. Phys. Rev. Lett. 1996, 77, $3865 ; 1997,78,1396$ (E) for other GGAs see references therein. 439.

(15) Rabuck, A. D.; Scuseria, G. E. Theor. Chem. Acc. 2000, 104,

(16) Staroverov, V. N.; Scuseria, G. E.; Perdew, J. P.; Tao, J.; Davidson, E. R. Phys. Rev. A 2005, 70, 012502.

(17) Perdew, J. P.; Tao, J.; Staroverov, V. N.; Scuseria, G. E. J. Chem. Phys. 2004, 120, 6898

(18) Csonka, G. I.; Ruzsinszky, A.; Tao, J.; Perdew, J. P. Int. J. Quantum Chem. 2005, 101, 506.

(19) Staroverov, V. N.; Scuseria, G. E.; Tao, J.; Perdew, J. P. Phys. Rev. B 2004, 69, 075102 .

(20) Furche, F.; Perdew, J. P. J. Chem. Phys., submitted.

(21) Ruzsinszky, A.; Perdew, J. P.; Csonka, G. I. J. Phys. Chem. A, in press (paper I).

(22) Johnson, E. R.; Wolkow, R. A.; DiLabio, G. A. Chem. Phys. Lett. 2004, 394, 334

(23) Johnson, E. R.; Wolkow, R. A.; DiLabio, G. A. Chem. Phys. Lett. 2005, 401, 595.

(24) Murray, C. W.; Handy, N. C.; Amos, R. D. J. Chem. Phys. 1993, $98,7195$.

(25) Kohn, A.; Weigend, F.; Alrichs, R. Phys. Chem. Chem. Phys. 2001, 3,711

(26) Jellinek, J.; Acioli, P. H. J. Phys. Chem. A 2002, 109, 436

(27) Sponer, J.; Jurecka, P.; Hobza, P. J. Am. Chem. Soc. 2004, 126 , 10142 .

(28) Zhao, Y.; Truhlar, D. G. J. Chem. Theory Comput. 2005, 1, 415.

(29) Zhao, Y.; Truhlar, D. G. J. Phys. Chem. A 2005, 109, 4209.

(30) Sinnokrot, M. O.; Sherrill, D. J. Phys. Chem. A 2004, 108, 1020010207.

(31) Csonka, G. I. Unpublished work, 2005.

(32) Becke, A. D. Phys. Rev. A 1988, 38, 3098.

(33) Wu, Q.; Yang, W. J. Chem. Phys. 2002, 116, 515.

(34) Lima, N. A.; Caldas, M. J. Phys Rev. B, to be published.

(35) Grimme, S. J. Comput. Chem. 2004, 25, 1463.

(36) Lilienfeld, O. A.; Tavernelli, I.; Rothlisberger, U.; Sebastiani, D. Phys. Rev. B 2005, 71, 195119 .

(37) Wu, X.; Vargas, M. C.; Nayak, S.; Lotrich, V.; Scoles, G. J. Chem. Phys. 2001, 115, 8748.
(38) Perdew, J. P. In Electronic Structure of Solids '91; Ziesche, P., Eschrig, H., Eds.; Akademie Verlag: Berlin, 1991; p 11.

(39) Feller, D. http://www.emsl.pnl.gov/forms/basisform.html (accessed Feb 2004).

(40) Frisch, M. J.; Trucks, G. W.; Schlegel, H. B.; Scuseria, G. E.; Robb, M. A.; Cheeseman, J. R.; Montgomery, J. A., Jr.; Vreven, T.; Kudin, K. N.; Burant, J. C.; Millam, J. M.; Iyengar, S. S.; Tomasi, J.; Barone, V.; Mennucci, B.; Cossi, M.; Scalmani, G.; Rega, N.; Petersson, G. A. Nakatsuji, H.; Hada, M.; Ehara, M.; Toyota, K.; Fukuda, R.; Hasegawa, J.; Ishida, M.; Nakajima, T.; Honda, Y.; Kitao, O.; Nakai, H.; Klene, M.; Li, X.; Knox, J. E.; Hratchian, H. P.; Cross, J. B.; Bakken, V.; Adamo, C.; Jaramillo, J.; Gomperts, R.; Stratmann, R. E.; Yazyev, O.; Austin, A. J.; Cammi, R.; Pomelli, C.; Ochterski, J. W.; Ayala, P. Y.; Morokuma, K.; Voth, G. A.; Salvador, P.; Dannenberg, J. J.; Zakrzewski, V. G.; Dapprich, S.; Daniels, A. D.; Strain, M. C.; Farkas, O.; Malick, D. K.; Rabuck, A D.; Raghavachari, K.; Foresman, J. B.; Ortiz, J. V.; Cui, Q.; Baboul, A. G.; Clifford, S.; Cioslowski, J.; Stefanov, B. B.; Liu, G.; Liashenko, A.; Piskorz, P.; Komaromi, I.; Martin, R. L.; Fox, D. J.; Keith, T.; Al-Laham, M. A.; Peng, C. Y.; Nanayakkara, A.; Challacombe, M.; Gill, P. M. W; Johnson, B.; Chen, W.; Wong, M. W.; Gonzalez, C.; Pople, J. A. Gaussian 03, revision C.01; Gaussian, Inc.: Wallingford, CT, 2004.

(41) Olgivie, J. F.; Wang, F. Y. H. J. Mol. Struct. 1992, 273, 277; 1993, 291,313 and references therein.

(42) Aziz, R. A.; Slaman, M. J. Chem. Phys. 1989, 130, 187.

(43) Aziz, R. A. J. Chem. Phys. 1993, 994158.

(44) Csonka, G. I. J. Mol. Struct. (THEOCHEM) 2002, 584, 1 and references therein.

(45) Xu, X.; Goddard, W. A., III. Proc. Natl. Acad. Sci. U.S.A. 2004, 101, 2673.

(46) Ernzerhof, M.; Scuseria, G. E. J. Chem. Phys. 1999, 110, 5029.

(47) Adamo, C.; Cossi, M.; Barone, V. J. Mol. Struct. (THEOCHEM) 1999, 493,145 .

(48) Tang, K. T.; Toennies, J. P. J. Chem. Phys. 2003, 118, 4976.

(49) Füsti-Molnár, L.; Szalay, P. G. Chem. Phys. Lett. 1996, 258, 400.

(50) Sosa, C.; Noga, J.; Bartlett, R. J. J. Chem. Phys. 1988, 88, 5974.

(51) Evangelisti, S.; Bendazzoli, G. L.; Gagliardi, L. Chem. Phys. 1994, 185,47

(52) Bondybey, V. E. Chem. Phys. Lett. 1984, 109, 436.

(53) Petterson, G. A.; Shirley, W. A. Chem. Phys. Lett. 1989, 160, 494. 